\title{
Vitrectomy in aphakia: a simplified technique for fundus visualisation*
}

\author{
OSAMA M ASFOUR AND AYMAN NASSAR
}

From Benha University, Egypt

SUMMARY By using a small air bubble that fills about two thirds of the anterior chamber the erect image of the fundus of the aphakic human eye can be visualised clearly during vitrectomy, enabling the surgeon to operate without the need for vitrectomy lenses. The refractive power of the human aphakic eye is calculated here and the image site and size are presented.

During vitreous surgery surgeons use different types of vitrectomy lenses that are either fixed to the corneosclera or held by an assistant to provide a clear view of the fundus. In patients undergoing vitrectomy the removal of the crystalline lens is rather common. In this paper we revive a simple old optical principle that we hope will simplify and shorten the vitrectomy techniques in aphakia.

In our aphakic patients undergoing vitrectomy and in patients undergoing pars plana lensectomy and vitrectomy we were able to provide a clear view of the fundus during surgery simply by injecting through a limbal incision a small air bubble that fills one-half to two-thirds of the anterior chamber. Owing to the bubble's small size and to the minimal movement of the eye during surgery the back of the cornea was kept wet all the time, leading to perfect visibility during the whole procedure. This obviates the fogging effect caused by irregular wetting of the back of the cornea when the whole eye is filled with gas or air. Peripheral portions of the fundus were easily visualised by tilting the globe in the desired direction, the examination being aided by the superior position of the air bubble in the anterior chamber. The change in magnification owing to the relative peripheral corneal flattening is gradual and practically negligible.

\section{Optics}

In aphakia the refraction of the eye occurs at the anterior and posterior surfaces of the cornea and is

\footnotetext{
*This article was presented to the 9th Afro-Asian Congress of
} Ophthalmology, Cairo, Egypt, on 23 February 1988.

Correspondence to Osama M Asfour, MD, 6 Aly Abi Talib Street, Mohandesin, Cairo, Egypt.
43.05 dioptres. ${ }^{1}$ The equivalent refractive power of two separated optical surfaces can be given by the Gullstrand's equation:

$$
F=D 1+D 2-D 1 D 2 * d / n,
$$

where $F$ is the total refractive power of the two surfaces, D1 and D2 are the refractive powers of the two surfaces, $d$ is the distance between the two refracting elements (measured in metres), and $\mathbf{n}$ is the refractive index of the medium in between. ${ }^{2}$ (The asterisk denotes multiplication.)

The optical power of the aphakic eye when $1 / 2$ to $2 / 3$ of anterior chamber volume is filled with air is nearly the same as when the whole eye is filled with air, that is, about -5.48 dioptres, owing to the relatively flat interface between the air bubble and aqueous or vitreous.

When the same refractive index is present on both sides of the lens, the nodal and principal points coincide. ${ }^{3}$ And since in that situation the cornea is the only refractive system and is surrounded by air on

\section{Table 1 From 'Schematic eye'2}

\begin{tabular}{lr}
\hline Refractive index & \\
Cornea & $1 \cdot 376$ \\
Aqueous humor and vitreous body & $1 \cdot 336$ \\
Position & 0 \\
Anterior surface of cornea & $0 \cdot 5$ \\
Posterior surface of cornea & $7 \cdot 2$ \\
Posterior surface of lens & \\
Radius of curvature & $7 \cdot 7$ \\
$\quad$ Anterior surface of cornea & $6 \cdot 8$ \\
Posterior surface of cornea & -6.0 \\
Posterior surface of lens & 48.83 \\
Refracting power & 43.05 \\
Anterior surface of cornea & \\
Corneal system & \\
\hline
\end{tabular}



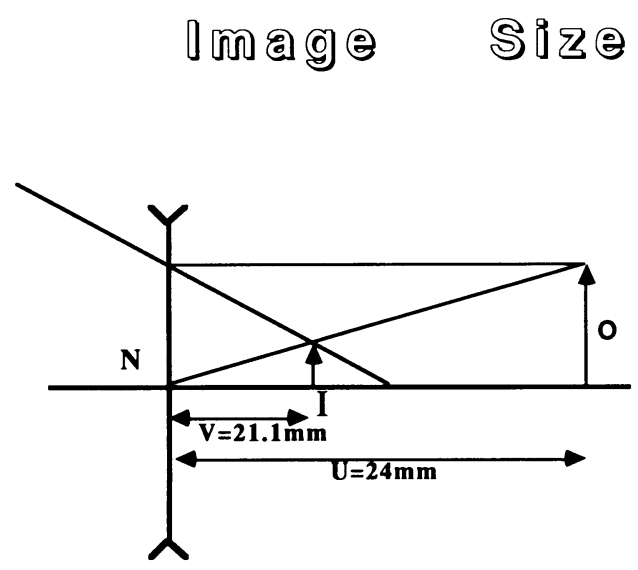

Image Size Change $=\frac{I}{0}=\frac{V}{U}=\frac{21.1}{24}=0.88$

Fig. 1 The imagelobject size ratio.

either side, all measurements were calculated from the anterior corneal surface.

As regards the position of the image, it was found to be situated about $21 \cdot 1 \mathrm{~mm}$ from the corneal surface, as calculated from the equation

$$
\begin{aligned}
& \mathrm{D}=1 / \mathrm{U}+1 / \mathrm{V} \\
& \mathrm{V}=1 /[\mathrm{D}-(1 / \mathrm{U})],
\end{aligned}
$$

where $\mathrm{D}$ is the refractive power of the cornea with air on both sides, and $U$ and $V$ are the corresponding retinal and image distances from the corneal surface.

The image/object size ratio as calculated from the following equation is about $0 \cdot 88$ :

$$
\text { Image/object }=\mathrm{I} / \mathrm{O}=\mathrm{V} / \mathrm{U}=21 \cdot 1 / 24=0 \cdot 88 \text {, }
$$

where $\mathrm{O}$ and $\mathrm{I}$ are the corresponding object and image sizes, and $\mathrm{V}$ and $\mathrm{U}$ are the corresponding image and retinal distances from the surface of the cornea.

This means that we get a slightly reduced virtual image of the retina that is situated inside the eye about $3 \mathrm{~mm}$ in front of an emmetropic retina.

\section{References}

1 Landers MB III, Stefansson E, Wolbarsht ML. The optics of vitreous surgery. Am J Ophthalmol 1981; 91: 611.

2 Helmholtz H. Treatise on physiological optics. New York: Dover, 1924: 1.

3 Obstfeld H. Optics in vision. London: Butterworths, 1982: 21.

Accepted for publication 28 April 1988. 